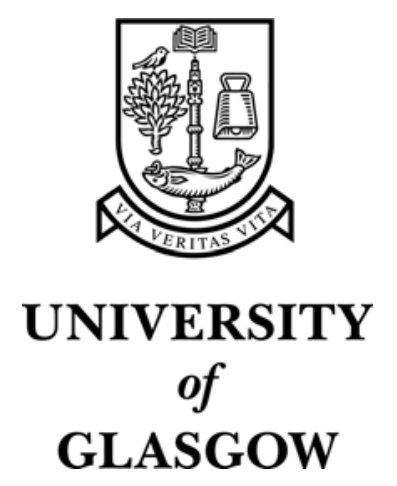

Paul, M.C. and Rees, D.A.S. and Wilson, M. (2005) Influence of higher order effects on the vortex instability of thermal boundary layer flow in a wedge shaped domain. International Journal of Heat and Mass Transfer 48(8):pp. 1417-1424.

http://eprints.gla.ac.uk/3328/ 


\title{
THE INFLUENCE OF HIGHER ORDER EFFECTS ON THE VORTEX INSTABILITY OF THERMAL BOUNDARY LAYER FLOW IN A WEDGE-SHAPED DOMAIN
}

\author{
Manosh C. Paul ${ }^{\dagger}$, D. Andrew S. Rees*, Michael Wilson \\ Department of Mechanical Engineering, University of Bath, \\ Bath, BA2 7AY, UK
}

\begin{abstract}
We reconsider the onset of streamwise vortices in the thermal boundary layer flow induced by an inclined upward-facing heated semi-infinite surface placed within a Newtonian fluid. Particular emphasis is laid upon how the induced flow in the isothermal region outside the boundary layer affects the boundary layer itself at higher order, and how this, in turn, affects the stability criterion for the onset of vortices. We find that the stability criterion for thermal boundary layers in air is less susceptible to changes in external geometry than for boundary layers in water. In general, we conclude that the variation of the stability criterion with wedge angle (between the heated and the outer boundary surface) is too great for the theory to predict reliably where disturbances first begin to grow.
\end{abstract}

Keywords: Free convection; Boundary layer; Vortex instability; Higher order effects

\section{Introduction.}

The general problem of stability of free convection boundary layer flows is a combination of the problems of hydrodynamic instability and thermo-convective instability. When an upward-facing semi-infinite heated surface is inclined from the horizontal and is such that the leading edge is below the rest of the surface, then resulting flow is thermo-convectively unstable since it is unstably stratified. In this regard it shares some characteristics with the classical Bénard problem which consists of a layer of fluid which is heated from below. In such cases the primary mode of instability takes the form of longitudinal vortices with axes aligned in the general direction of the induced basic flow.

When the heated surface is vertical buoyancy forces generate the basic flow directly, but do not cause its destabilisation. Since the maximum streamwise velocity increases as $x^{1 / 2}$ and the boundary layer thickness as $x^{1 / 4}$, a local Reynolds number based on these quantities increases as the $\frac{3}{4}$ power of the distance from the leading edge, and thus it may be suspected strongly that instabilities are hydrodynamic in nature. Some confirmation

$\dagger$ Current address: Department of Mechanical Engineering, University of Glasgow, Glasgow G12 8QQ, Scotland

* Corresponding author. Email: D.A.S.Rees@bath.ac.uk 
of this suspicion is provided by the identity of the primary mode of instability, namely, two-dimensional travelling waves, for these also form the primary mode for the isothermal Blasius boundary layer. Additionally, waves also arise when the heated surface is downward-facing, for which the boundary layer is stably stratified.

Thus, as the inclination of an upward-facing heated surface approaches the vertical the destabilizing influence of the unstable temperature gradient wanes, but the direct action of buoyancy forces increases and this increases the destabilizing influence of the magnitude of the streamwise velocity. Therefore there is a corresponding changeover between the identity of the most unstable mode as the inclination increases, and this has been verified by the experiments of Lloyd and Sparrow [1]. Using water as the working fluid they found that waves provide the dominant mode of instability when the inclination from the vertical is less than $14^{\circ}$, and vortices when the inclination is greater than $17^{\circ}$. At intermediate angles waves and vortices seem to coexist. On the theoretical side, which comprises only linear stability analyses to date (with one exception, see Chen et al [2]), Iyer and Kelly [3], using the parallel flow approximation, found that both modes become linearly unstable at the same downstream location at an inclination from the vertical of only 4 degrees. They argue that this result is consistent with the experimental work when account is taken of modal amplification rates. Both modes individually are sufficiently strong to be first observed at the same streamwise location when the inclination is 17 degrees.

In this paper we do not enter into the debate over the circumstances in which vortices are more or less important than waves. But rather we are concerned with the effect on the vortex stability criterion of the induced flow which is external to the boundary layer itself. Haaland and Sparrow [4], Chen and Tzuoo [5], and Kahawita and Meroney [6] used the leading order boundary layer flow as the basic flow to analyse stability. Thus their analyses take no account of the overall shape of the flow domain.

Here the fluid region is bounded by two semi-infinite flat plates forming a wedge of angle $\alpha$; this serves as a convenient way of analysing the influence of the shape of the fluid domain on the stability criterion. The heated, constant temperature plate is inclined at an angle $\delta$ from the vertical, while the other plate is either insulated or is held at the ambient temperature of the fluid. The basic flow used in the analysis is a two-term boundary layer approximation using the method of matched asymptotic expansions, the second term of which depends on the wedge angle. Such a technique has been used recently by Paul et al [7] and Storesletten and Rees [8]. The former authors considered the wave instability for a vertical heated surface, while the latter considered vortex instabilities in thermal boundary layer flows in porous media. We find that the critical distance from the leading edge beyond which disturbances grow is dependent on both $\delta$ and $\alpha$, and this suggests that the external geometry of the fluid domain does exert an influence on stability criteria. We consider two common fluids: air (for which $\operatorname{Pr}=0.7)$ and water $(\operatorname{Pr}=6.7)$. 


\section{Nomenclature}

\section{$d \quad$ dimensional length scale}

$F, G, H, T$ disturbances

$f_{0}, h_{0} \quad$ leading order boundary layer solutions

$f_{1}, h_{1} \quad$ first order boundary layer solutions

$g \quad$ gravitational acceleration

$k \quad$ wavenumber

$p \quad$ dynamic pressure

Pr Prandtl number

$t \quad$ time

$u, v, w \quad$ fluid velocities in the $x, y$, and $z$ directions

$U \quad$ dimensional velocity scale

$x, y, z \quad$ Cartesian coordinates

Greek symbols

$\begin{array}{ll}\alpha & \text { wedge angle } \\ \beta & \text { coefficient of cubical expansion } \\ \delta & \text { surface inclination angle } \\ \Delta T & \text { dimensional temperature range } \\ \epsilon & \text { small value } \\ \eta & \text { similarity variable } \\ \mu & \text { dynamic viscosity } \\ \theta & \text { temperature function } \\ \phi & \text { angular coordinate } \\ \psi & \text { streamfunction } \\ \xi & \text { scaled } x \text {-variable } \\ \omega & \text { vorticity }\end{array}$

Superscripts and subscripts

$\begin{array}{ll}c & \text { critical } \\ n & \text { normal derivative } \\ \infty & \text { ambient conditions } \\ , & \text { derivatives with respect to } \eta \\ - & \text { basic flow quantities } \\ & \text { disturbance quantities }\end{array}$

In $\S 2$ we derive the governing equations for thermal boundary layer flow from an inclined surface. A basic flow analysis is presented in $\S 3$ using matched aysmptotic expansions. The equations satisfied by the vortex disturbances are derived in $\S 4$ using a linearised analysis. The numerical techniques used are discussed in $\S 5$ and the results are presented in $\S 6$. We summarise our conclusions in $\S 7$.

\section{Governing equations of motion.}

We consider the instability of free convective boundary layer flow from an inclined semi-infinite heated plate. A sketch of the flow configuration is shown in Fig. 1. The 
equations which describe the free convection flow are taken to be the Navier-Stokes and energy equations. The unsteady equations of motion, which are subject to the Boussinesq approximation, are written in the following nondimensional form,

$$
\begin{gathered}
\frac{\partial u}{\partial x}+\frac{\partial v}{\partial y}+\frac{\partial w}{\partial z}=0 \\
\frac{\partial u}{\partial t}+u \frac{\partial u}{\partial x}+v \frac{\partial u}{\partial y}+w \frac{\partial u}{\partial z}=-\frac{\partial p}{\partial x}+\frac{\partial^{2} u}{\partial x^{2}}+\frac{\partial^{2} u}{\partial y^{2}}+\frac{\partial^{2} u}{\partial z^{2}}+\theta \cos \delta \\
\frac{\partial v}{\partial t}+u \frac{\partial v}{\partial x}+v \frac{\partial v}{\partial y}+w \frac{\partial v}{\partial z}=-\frac{\partial p}{\partial y}+\frac{\partial^{2} v}{\partial x^{2}}+\frac{\partial^{2} v}{\partial y^{2}}+\frac{\partial^{2} v}{\partial z^{2}}+\theta \sin \delta \\
\frac{\partial w}{\partial t}+u \frac{\partial w}{\partial x}+v \frac{\partial w}{\partial y}+w \frac{\partial w}{\partial z}=-\frac{\partial p}{\partial z}+\frac{\partial^{2} w}{\partial x^{2}}+\frac{\partial^{2} w}{\partial y^{2}}+\frac{\partial^{2} w}{\partial z^{2}} \\
\frac{\partial \theta}{\partial t}+u \frac{\partial \theta}{\partial x}+v \frac{\partial \theta}{\partial y}+w \frac{\partial \theta}{\partial z}=\operatorname{Pr}^{-1}\left(\frac{\partial^{2} \theta}{\partial x^{2}}+\frac{\partial^{2} \theta}{\partial y^{2}}+\frac{\partial^{2} \theta}{\partial z^{2}}\right)
\end{gathered}
$$

Here $x, y$ and $z$ are the streamwise, cross-stream and spanwise Cartesian coordinates, and $u, v$ and $w$ are the corresponding fluid velocity components. Further, $p$ is the dynamic pressure, $\theta$ is the fluid temperature, $t$ is time, and $\operatorname{Pr}$ is the Prandtl number. In eqs. (1) the angle of inclination of the semi-infinite surface from the vertical is $\delta$, where $0<\delta<\pi / 2$ corresponds to an upward facing surface with the leading edge placed vertically below the rest of the surface; see Fig. 1. We do not include the extreme cases, $\delta=0$ and $\delta=\pi / 2$ in our study because waves (rather than vortices) form the primary instability mechanism in the former case, while the similarity variable takes a different form in the latter case. Eqs. (1) have been nondimensionalised using

$$
d=\left(\frac{\nu^{2}}{g \beta \Delta T}\right)^{1 / 3} \quad \text { and } \quad U=(g \beta \Delta T \nu)^{1 / 3}
$$

as natural length and velocity scales. The ramification of using these scales is that the Grashof number has effectively been set to unity. A similar procedure has also been used by Paul et al [7] for the wave instability of the vertical boundary layer (i.e. for $\delta=0$ ), and by Rees $[9,10]$ in studies of vortex disturbances in thermal boundary layer flows in porous media.

The surface $y=0, x \geq 0$, is isothermal and is maintained at the temperature $\theta=1$, while the other bounding surface is either maintained at the ambient temperature, $\theta=0$, or else it is insulated. The corresponding boundary conditions may be written down as follows

$$
\begin{gathered}
u=v=w=0, \quad \theta=1 \quad \text { at } \quad y=0, \quad x \geq 0, \quad \text { on } \quad \phi=0, \\
u=v=w=0, \quad \theta=0 \text { or } \theta_{n}=0 \quad \text { on } \quad \phi=\alpha .
\end{gathered}
$$

In (3) $\phi$ measures the angular coordinate relative to the heated surface. 


\section{Basic flow analysis.}

We now determine the two-term solution of the undisturbed basic boundary layer flow using the method of matched asymptotic expansions. The basic steady flow, whose stability is being considered, is two-dimensional and may be written in a streamfunctionvorticity form. We may assume that all $z$ and $t$ derivatives are zero and therefore we substitute $u=\bar{\psi}_{y}$ and $v=-\bar{\psi}_{x}$ into Eqs. (1) and (3). The following equations for the basic flow are obtained,

$$
\begin{gathered}
\nabla^{2} \bar{\psi}=\bar{\omega} \\
\nabla^{2} \bar{\omega}=\frac{\partial \bar{\psi}}{\partial y} \nabla^{2}\left(\frac{\partial \bar{\psi}}{\partial x}\right)-\frac{\partial \bar{\psi}}{\partial x} \nabla^{2}\left(\frac{\partial \bar{\psi}}{\partial y}\right)+\frac{\partial \bar{\theta}}{\partial y} \cos \delta-\frac{\partial \bar{\theta}}{\partial x} \sin \delta \\
\operatorname{Pr}^{-1} \nabla^{2} \bar{\theta}=\frac{\partial \bar{\psi}}{\partial y} \frac{\partial \bar{\theta}}{\partial x}-\frac{\partial \bar{\psi}}{\partial x} \frac{\partial \bar{\theta}}{\partial y}
\end{gathered}
$$

which are to be solved subject to the boundary conditions,

$$
\begin{gathered}
\bar{\psi}=\frac{\partial \bar{\psi}}{\partial y}=0, \quad \bar{\theta}=1, \quad \text { on } \quad y=0(\phi=0), \\
\bar{\psi}=0, \quad \bar{\theta}=0 \text { or } \frac{\partial \bar{\theta}}{\partial n}, \quad \text { on } \quad \phi=\alpha .
\end{gathered}
$$

We have denoted the basic flow by overbars.

The leading order boundary layer flow now follows simply by assuming that $x \gg y$ and neglecting the streamwise diffusion terms. This boundary layer flow entrains isothermal fluid from the region outside the layer, but the detailed external flowfield which is produced depends on the shape of the domain. Thus the boundary layer itself is modified (albeit slightly when $x \gg y$ ) by this external flow. Our aim here is to determine the second term in the boundary layer expansion and to use it as part of the basic flow in a vortex stability analysis.

In the boundary layer region (i.e. where $x \gg y$ ) we may expand the streamfunction and temperature in terms of the following series (see in Hieber [11] for a case of $\delta=0$ ),

$$
\begin{gathered}
\bar{\psi}=x^{3 / 4}(\cos \delta)^{1 / 4}\left[f_{0}(\eta)+x^{-3 / 4} f_{1}(\eta)+\cdots \cdots\right] \\
\bar{\theta}=h_{0}(\eta)+x^{-3 / 4} h_{1}(\eta)+\cdots \cdots
\end{gathered}
$$

where the similarity variable $\eta$ is given by

$$
\eta=y(\cos \delta)^{1 / 4} x^{-1 / 4}
$$

The functions $f_{0}, f_{1}, h_{0}$, and $h_{1}$ which appear in Eq. (5) satisfy the following ordinary differential equations where primes represent derivatives with respect to $\eta$,

$$
f_{0}^{\prime \prime \prime}+\frac{3}{4} f_{0} f_{0}^{\prime \prime}-\frac{1}{2} f_{0}^{\prime} f_{0}^{\prime}+h_{0}=0
$$




$$
\begin{gathered}
h_{0}^{\prime \prime}+\frac{3}{4} \operatorname{Pr} f_{0} h_{0}^{\prime}=0, \\
f_{1}^{\prime \prime \prime}+\frac{3}{4} f_{0} f_{1}^{\prime \prime}-\frac{1}{4} f_{0}^{\prime} f_{1}^{\prime}+h_{1}=\frac{1}{4} \tan \delta(\cos \delta)^{-1 / 4}\left(\eta h_{0}-\int_{0}^{\eta} h_{0}(\xi) d \xi\right), \\
h_{1}^{\prime \prime}+\frac{3}{4} \operatorname{Pr}\left(f_{0} h_{1}\right)^{\prime}=0 .
\end{gathered}
$$

At $\eta=0$ these functions satisfy the following boundary conditions

$$
\begin{gathered}
f_{0}=f_{0}^{\prime}=f_{1}=f_{1}^{\prime}=0, \\
h_{0}=1, \quad h_{1}=0,
\end{gathered}
$$

whilst the appropriate boundary conditions which match with the outer flow (as $\eta \rightarrow \infty)$ are that

$$
\begin{gathered}
f_{0}^{\prime} \rightarrow 0, \quad f_{1}^{\prime} \rightarrow-\frac{3}{4} f_{0}(\infty)(\cos \delta)^{-1 / 4} \cot \left(\frac{3}{4} \alpha\right), \\
h_{0} \rightarrow 0, \quad h_{1} \rightarrow 0
\end{gathered}
$$

The matched asymptotic analysis which gives rise to the condition (8c) is almost identical to that presented in [7] and is omitted here.

The system of Eqs. (7a)-(7d) must be solved numerically and this is discussed in $\S 5$. We note that the definition of $\eta$ which has been used means that the leading order boundary layer flow is independent of $\delta$, but the second order terms are functions of both $\alpha$ and $\delta$.

\section{Linear stability analysis.}

In this section we develop the linear stability equations for the basic flow given above. It is well-known that the primary mode of instability for an upward facing inclined hot surfaces takes the form of streamwise vortices when the heated surface is not too close to the vertical; see [1]. Therefore we will consider small disturbances of this form.

We perturb the basic solutions by setting

$$
(u, v, w, \theta, p)=(\bar{u}, \bar{v}, 0, \bar{\theta}, \bar{p})+\epsilon(\hat{u}, \hat{v}, \hat{w}, \hat{\theta}, \hat{p})
$$

where $\hat{u}, \hat{v}, \hat{w}, \hat{\theta}$, and $\hat{p}$ are the components of the disturbance and are functions of $x, y$, $z$, and $t$. The disturbance amplitude, $\epsilon$, is assumed to be infinitesimal. Substitution of Eq. (9) into the full governing Eqs. (1), followed by linearisation yields the following set of disturbance equations,

$$
\begin{gathered}
\hat{u}_{x}+\hat{v}_{y}+\hat{w}_{z}=0, \\
\hat{u}_{t}+\bar{u} \hat{u}_{x}+\bar{u}_{x} \hat{u}+\bar{v} \hat{u}_{y}+\bar{u}_{y} \hat{v}=-\hat{p}_{x}+\hat{u}_{x x}+\hat{u}_{y y}+\hat{u}_{z z}+\hat{\theta} \cos \delta, \\
\hat{v}_{t}+\bar{u} \hat{v}_{x}+\bar{v}_{x} \hat{u}+\bar{v} \hat{v}_{y}+\bar{v}_{y} \hat{v}=-\hat{p}_{y}+\hat{v}_{x x}+\hat{v}_{y y}+\hat{v}_{z z}+\hat{\theta} \sin \delta,
\end{gathered}
$$




$$
\begin{gathered}
\hat{w}_{t}+\bar{u} \hat{w}_{x}+\bar{v} \hat{w}_{y}=-\hat{p}_{z}+\hat{w}_{x x}+\hat{w}_{y y}+\hat{w}_{z z}, \\
\hat{\theta}_{t}+\bar{u} \hat{\theta}_{x}+\bar{\theta}_{x} \hat{u}+\bar{v} \hat{\theta}_{y}+\bar{\theta}_{y} \hat{v}=\operatorname{Pr}^{-1}\left(\hat{\theta}_{x x}+\hat{\theta}_{y y}+\hat{\theta}_{z z}\right) .
\end{gathered}
$$

The appropriate boundary conditions are

$$
\begin{gathered}
\hat{u}=\hat{v}=\hat{w}=\hat{\theta}=0, \quad \text { at } \quad y=0, \text { for } x>0, \\
\hat{u}, \hat{v}, \hat{w}, \hat{\theta}, \rightarrow 0, \quad \text { as } \quad y \rightarrow \infty \text { for } x>0 .
\end{gathered}
$$

We note that, strictly speaking, y cannot increase indefinitely whenever the wedge angle is less than $\pi / 2$. However, it is sufficient that the thermal boundary layer is much narrower than the perpendicular distance from the heated surface to the second surface, and, in general, this means that our analysis usually remains valid for fairly small values of $\alpha$.

We now make the same assumption as [4-6] and assume that marginal stability corresponds to where there is no streamwise variation with $x$, and that the onset of vortices is stationary in time. Therefore, we assume that the vortices are functions solely of $y$ and $z$, and that they are periodic in the spanwise direction $z$. Consequently, the disturbance quantities $\hat{u}, \hat{v}, \hat{w}, \hat{\theta}$, and $\hat{p}$ may be Fourier decomposed using

$$
\begin{gathered}
\hat{u}=F(y) \cos k z, \quad \hat{v}=G(y) \cos k z, \\
\hat{w}=H(y) \sin k z, \quad \hat{\theta}=T(y) \cos k z, \quad \hat{p}=Q(y) \cos k z,
\end{gathered}
$$

where $F(y), G(y), H(y), T(y)$, and $Q(y)$ are small amplitude functions and $k$ is the spanwise wavenumber of the disturbances. Here the temporal growth rate is considered to be zero to correspond to neutral stability.

Eqs. (12) may now be substituted into Eqs. (10). For computational convenience, we eliminate $Q$ and $H$ which leads to three equations involving only $F, G$, and $T$. We also make the change of variables from $(x, y)$ to $(x, \eta)$ where the similarity variable $\eta$ is defined in Eq. (6). The disturbance equations now become

$$
\begin{gathered}
F^{\prime \prime}-\bar{v} \xi F^{\prime}-\left(k^{2}+\bar{u}_{x}\right) \xi^{2} F=\xi^{2}\left(\bar{u}_{y} G-T \cos \delta\right), \\
G^{i v}-\bar{v} \xi G^{\prime \prime \prime}-\left(2 K^{2}+\bar{v}_{y}\right) \xi^{2} G^{\prime \prime}+k^{2} \bar{v} \xi^{3} G^{\prime}+k^{2}\left(k^{2}+\bar{v}_{y}\right) \xi^{4} G=-k^{2} \bar{v}_{x} \xi^{4} F+k^{2} \xi^{4} T \sin \delta, \\
T^{\prime \prime}-\operatorname{Pr} \bar{v} \xi T-k^{2} \xi^{2} T=\operatorname{Pr} \xi^{2}\left(\bar{\theta}_{x} F+\bar{\theta}_{y} G\right),
\end{gathered}
$$

where the function, $\xi$, is defined as

$$
\xi(x, \delta)=\left(\frac{x}{\cos \delta}\right)^{1 / 4},
$$

The corresponding boundary conditions to be satisfied by the disturbance equations are that

$$
F=G=G^{\prime}=T=0, \quad \text { at } \quad \eta=0,
$$




$$
F=G=G^{\prime}=T=0, \quad \text { as } \quad \eta \rightarrow \infty
$$

The system of Eqs. (13)-(15) is homogeneous and therefore it is always satisfied by a zero solution. A normalisation condition is required to force nonzero solutions, and this is taken to be

$$
T^{\prime}(0)=1
$$

Eqs. (15) and (16) form nine boundary conditions for the eight order system of Eqs. (13). Therefore, one of the two parameters, $k$ and $x$ has to be chosen as the eigenvalue. However, in general it is found that the neutral stability curve has one well-defined minimum, and therefore it is this value which is of primary interest since it yields both the minimum distance beyond which vortex disturbances grow and the corresponding wavenumber. These values are computed by solving the system (13) to (16) together with the system obtained by differentiating each equation in (13) to (16) with respect to $k$ where $\partial \xi / \partial k=0$ defines the minimum of the neutral curve. This new extended system forms a 16th order system with 18 boundary conditions and two eigenvalues: $\xi$ and $k$. A suitably modified version of the Keller-box method is employed to solve this ordinary differential eigensystem and is discussed in the next section.

\section{Numerical method.}

The equations for the basic flow were solved using a standard shooting method which employs Newton-Raphson iteration techniques. In this method the ordinary differential Eqs. (7a,b,c,d) are written as a first order system of ordinary differential equations and a 4th order Runge-Kutta method employed to solve them. These solutions are accurate to at least six significant figures.

A modified version of the Keller-box code was employed to solve the disturbance equations. For this type of method it is not necessary always to insist that the governing equations are reduced to first order form, and the present code solves the second order differential equations using straightforward second order accurate central difference approximations. When the difference equations are suitably arranged the presence of two eigenvalues, $x$ and $k$ causes the Jacobian matrix of the Newton-Raphson iteration scheme, which is a central part of the Keller box methodology, to have two extra rows and columns over and above its usual block tridiagonal structure. Therefore the blockThomas algorithm is modified to account for this structural change; very similar schemes were used by Lewis et al [12] and Shu and Wilkes [13].

In addition, we have used $\eta_{\infty}=10$ with $d \eta=0.1$ for $\operatorname{Pr}=0.7$ and 6.7 and these were found to yield sufficiently accurate computations for both the main flow and the vortex disturbances. 


\section{Numerical results.}

In this section we present details of the vortex stability criterion and how this varies with both inclination angle and wedge angle. We have scaled the critical distance $x_{c}$ with respect to the inclination $\delta\left(0^{0}<\delta<90^{\circ}\right)$ angle according to

$$
\hat{x}_{c}=x_{c} \delta^{4 / 3},
$$

since the critical distance is proportional to $\delta^{-4 / 3}$ in the small- $\delta$ or vertical limit. Therefore we obtain a finite value for $\hat{x}_{c}$ as $\delta \rightarrow 0$. A similar type of scaling was used by Storesletten and Rees [8], and Hsu and Cheng [14] to analyse vortex instabilities in porous media, although the power of $\delta$ used in those studies is different.

Before presenting the detailed results of our stability analysis it is necessary to consider first some of the implications of the dependence of $F_{1}$ on $\alpha$. One component of the solution for $F_{1}$ is exactly proportional to $\cot \frac{3}{4} \alpha$; see the boundary condition (8c). Although this function is zero when $\alpha=\frac{2}{3} \pi=120^{\circ}$, the two-term boundary layer solution is not identical to the one-term solution since there is a second component of $F_{1}$ which corresponds to the inhomogeneous term in $(7 \mathrm{c})$. Therefore two-term stability results when $\alpha=\frac{2}{3} \pi$ will not be identical to those obtained using only the one-term solution. This is unlike the case considered by Paul et al [7] where $F_{1}$ is proportional to $\cot \frac{3}{4} \alpha$. When $\alpha=0$ and $\alpha=\frac{4}{3} \pi=240^{\circ} F_{1}$ is infinite and the asymptotic series breaks down in these limits. Furthermore, the function $\cot \frac{3}{4} \alpha$ has period $\frac{4}{3} \pi$ and therefore all the results for the range $0<\alpha \leq \frac{2}{3} \pi$ also apply for the range $\frac{4}{3} \pi<\alpha \leq 2 \pi$. Finally we note that $F_{1}$ is positive when $\frac{2}{3} \pi \leq \alpha<\frac{4}{3} \pi$, and therefore the two-term streamwise velocity is greater than that given by the leading term only. For other values of $\alpha$ the basic flow is slower.

First we will present the stability characteristics for air by choosing the Prandtl number to be 0.7. These results are summarised in Figs. 2, 3 and 4. Fig. 2 displays the variation of the scaled critical distance $\hat{x}_{c}$ with the inclination angle, $\delta$, for a set of discrete values of the wedge angle values in the range $5^{0} \leq \alpha \leq 235^{0}$. We note that the dashed curve corresponds to taking only the leading order boundary layer as the basic flow. At moderate inclinations of the surface we see that there is an extremely large variation in the value of $\hat{x}_{c}$ with $\alpha$, and therefore we may conclude immediately that stability criteria based on the leading order boundary layer theory gives misleading results, for the external domain exerts a very large influence on the stability characteristics of the flow.

However, when the heated surface is close to the vertical, i.e. $\delta$ is small, then there appears to be much less variation in the critical distance, $\hat{x}_{c}$. This is understandable since the unscaled value, $x_{c}$, is asymptotically large as $\delta \rightarrow 0$, and therefore the second term in the boundary layer expansion is asymptotically small compared with the leading term. Thus there should be little variation in $\hat{x}_{c}$ with $\alpha$. An alternative view of the stability characteristics may be seen in Fig. 3 wherein is displayed the variation of $\hat{x}_{c}$ with $\alpha$ for 
discrete values of $\delta$. In this Figure we see that the critical distance is essentially constant except when $\alpha$ is close to those values for which $\cot \frac{4}{3} \alpha$ is infinite (i.e. for $\alpha=0$ and $\alpha=120^{\circ}$ ). Therefore we conclude that we have some measure of confidence in the stability results at small inclinations, although we note that it is within this regime that waves are more likely to appear.

The critical wavenumber $\left(k_{c}\right)$ variation with $\delta$ is plotted in Fig. 4 . We see that there is little variation in $k_{c}$ as the wedge angle $\alpha$ varies, despite the large changes already observed in $\hat{x}_{c}$. But there is a distinct trend that the wavenumber decreases as $\delta$ decreases towards zero. This may be understood easily for the boundary layer thickens as $\delta \rightarrow 0$, and since the vortices tend to maintain a wavelength which is comparable to the local boundary layer thickness at onset, the wavenumber decreases towards zero as $x_{c} \rightarrow \infty$.

Equivalent results for the case of water, for which $\operatorname{Pr}=6.7$, are shown in Figs. 5 to 7. In general the critical distance for water is less than for air, but there appears to be slightly less variation in $\hat{x}_{c}$ with $\alpha$. Fig. 6 also indicates that the neutral distances vary less with $\alpha$ than they do for air. Therefore a little more confidence may be gained in using these results. The same trend is shown by the wavenumber as $\delta$ decreases towards zero, but the variation with $\alpha$ is greater than that for air. Therefore we have a somewhat conflicting picture, namely that the computed neutral distances are more reliable for water than for air, but that the wavenumber shows much more variation with wedge angle for air than for water.

Some comparison with published works may now be made, although very few published experimental papers deal with convection from a uniform temperature heated surface, with the rest concentrating on uniform heat flux surfaces. Both Lloyd and Sparrow [1] and Zuercher et al [15] have undertaken careful experimental studies of the onset of vortex convection in water. Estimates for the critical distance for the onset of vortices relies on being able to see disturbances, and these works yield 121 and 214 as the critical distance, $x_{c}$, at $\delta=45^{\circ}$. Both these values are well above the critical distances shown in Fig. 6. That this is so is not surprising for growing disturbances must achieve a certain threshold amplitude before being visable, as commented in [15]. However, Zuercher et al [15] also discuss why their results differ so much from those of Lloyd and Sparrow [1], concluding that the detailed configuration of the leading edge of the experiments might be an important factor. This reason is of the same type as that comprising the present paper, namely that the shape of the domain can and does exert a significant influence on the stability charateristics.

\section{Conclusions.}


We have used the theory of matched asymptotic expansions to determine a two-term approximation to the basic free convective boundary layer flow which is induced by an inclined heated surface. This flow has been analysed for stability with respect to vortex disturbances using the parallel flow approximation. The aim has been to determine what influence a more accurate representation of the basic flow has on the computed stability criteria.

Although the critical distance $\hat{x}_{c}$ is strongly dependent on the angle of inclination $\delta$, we have found that it also varies substantially with changes in the wedge angle $\alpha$. In this regard we may say that stability criteria derived using the leading order boundary layer flow are unreliable in general since the basic flow is clearly inadequately represented using one term. However, the presence of the second term in the basic flow yields stability criteria which are dependent on the overall shape of the convective domain. The only exception to this situation is when the heated surface is close to the vertical, for it is only in this limit that the second boundary layer terms are small compared with the leading order terms, and therefore the leading order criterion is accurate. However, it is in this regime that wave-like instabilities become more important than vortices.

\section{Acknowledgements}

The first author acknowledges gratefully the receipt of an Overseas Research Studentship (ORS) and a University of Bath Studentship which has supported the present work.

\section{References}

[1] J.R. Lloyd, E.M. Sparrow, On the instability of natural convection flow on inclined plates, J. Fluid Mech. 42 (3) (1970) 465-470.

[2] C.C. Chen, A. Labhari, H.-C. Chang, R.E. Kelly, Spanwise pairing of finite-amplitude vortex rolls in inclined free-convection boundary layers, J. Fluid Mech. 231 (1991) 73113.

[3] P.A. Iyer, R.E. Kelly, The stability of laminar free-convection flow induced by a heated inclined plate, Int. J. Heat Mass Transfer 17 (1974) 517-525.

[4] S.E. Haaland, E.M. Sparrow, Vortex instability of natural convection flow on inclined surfaces, Int. J. Heat Mass Transfer 16 (1973) 2355-2367.

[5] T.S. Chen, K.L. Tzuoo, Vortex instability of free convection flow over horizontal and inclined surfaces, J. Heat Transfer 104 (1982) 637-543.

[6] A. Kahawita, R.N. Meroney, The vortex mode of instability in natural convection flow along inclined plates, Int. J. Heat Mass Transfer 17 (1974) 541-548.

[7] M.C. Paul, D.A.S. Rees, M. Wilson, The influence of higher order effects on the linear 
wave instabilty of vertical free convective boundary layer flow, Submitted to Int. J. Heat Mass Transfer (2002).

[8] L. Storesletten, D.A.S. Rees, The influence of higher-order effects on the linear instability of thermal boundary layer flow in porous media, Int. J. Heat Mass Transfer 41 (1998) 1833-1843.

[9] D.A.S. Rees, Vortex instability from a near-vertical heated surface in a porous medium. I Linear theory, Proc. Roy. Soc. A 457 (2001) 1721-1734.

[10] D.A.S. Rees, Vortex instability from a near-vertical heated surface in a porous medium. II Nonlinear evolution, Proc. Roy. Soc. A 458 (2002) 1575-1592.

[11] C.A. Hieber, Natural convection around a semi-infinite vertical plate: Higher-order effects, Int. J. Heat Mass Transfer 17 (1971) 785-791.

[12] S. Lewis, A.P. Bassom, D.A.S. Rees, The stability of vertical thermal boundary layer flow in a porous medium, Eur. J. Mech. B 14 (1995) 395-408.

[13] J.J. Shu, G. Wilkes, Mixed convection laminar film condensation on a semi-infinite vertical plate, J. Fluid Mech. 300 (1995) 207-229.

[14] C.T. Hsu, P. Cheng, Vortex instability in buoyancy-induced flow over inclined heated surfaces in porous media, Trans. A.S.M.E. J. Fluid Engineering 101 (1979) 660-665.

[15] E.J. Zuercher, J.W. Jacobs, C.F. Chen, Experimental study of the stability of boundary-layer flow along a heated inclined plate, J. Fluid Mech. 367 (1998) 1-25. 


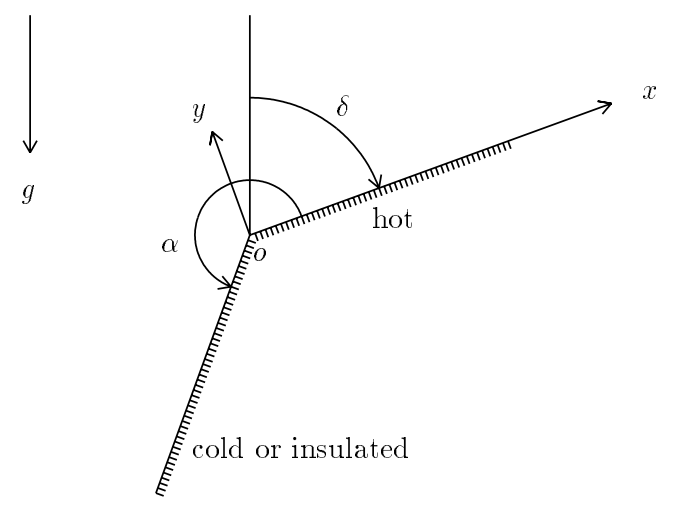

Fig. 1 .

The sketch of flow domain and coordinate system displaying wedge angle $(\alpha)$ and inclination angle $(\delta)$. 


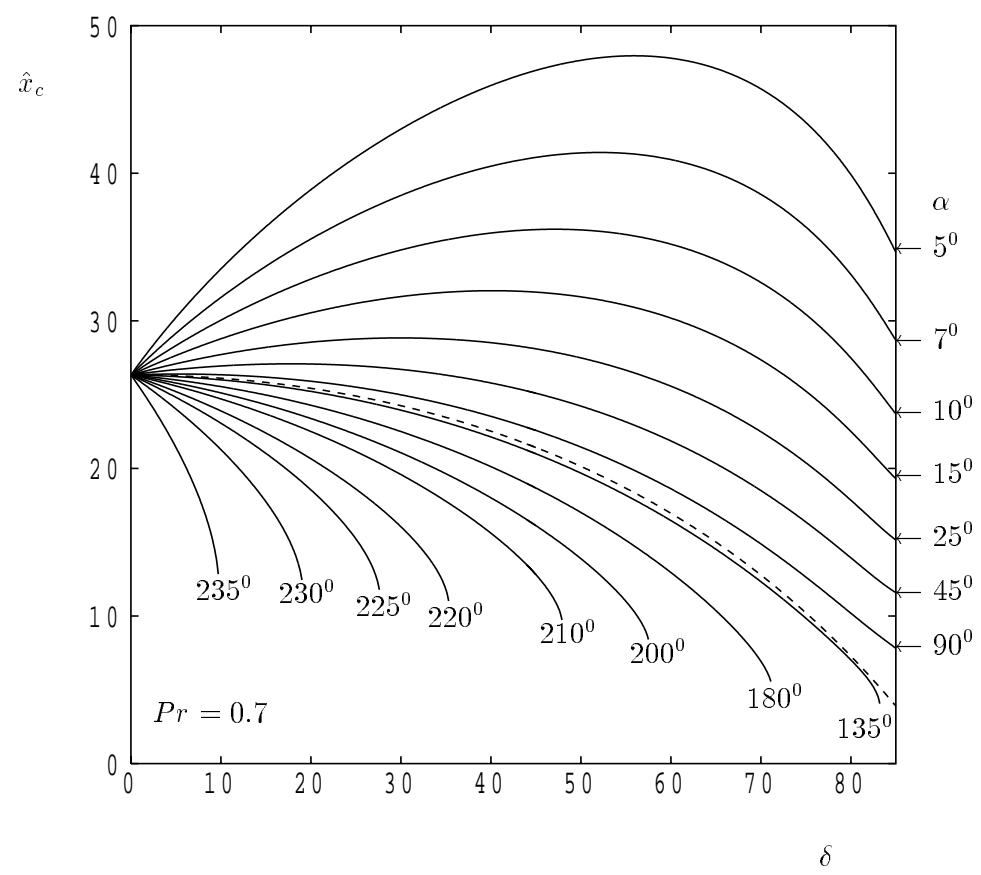

Fig. 2.

The variation of critical distance $\hat{x}_{c}$ with inclination angle $\delta$ for air $(\operatorname{Pr}=0.7)$ and $\alpha=5^{0}$ to $235^{0}$. The dashed line denotes the corresponding result for the leading order boundary layer flow. 


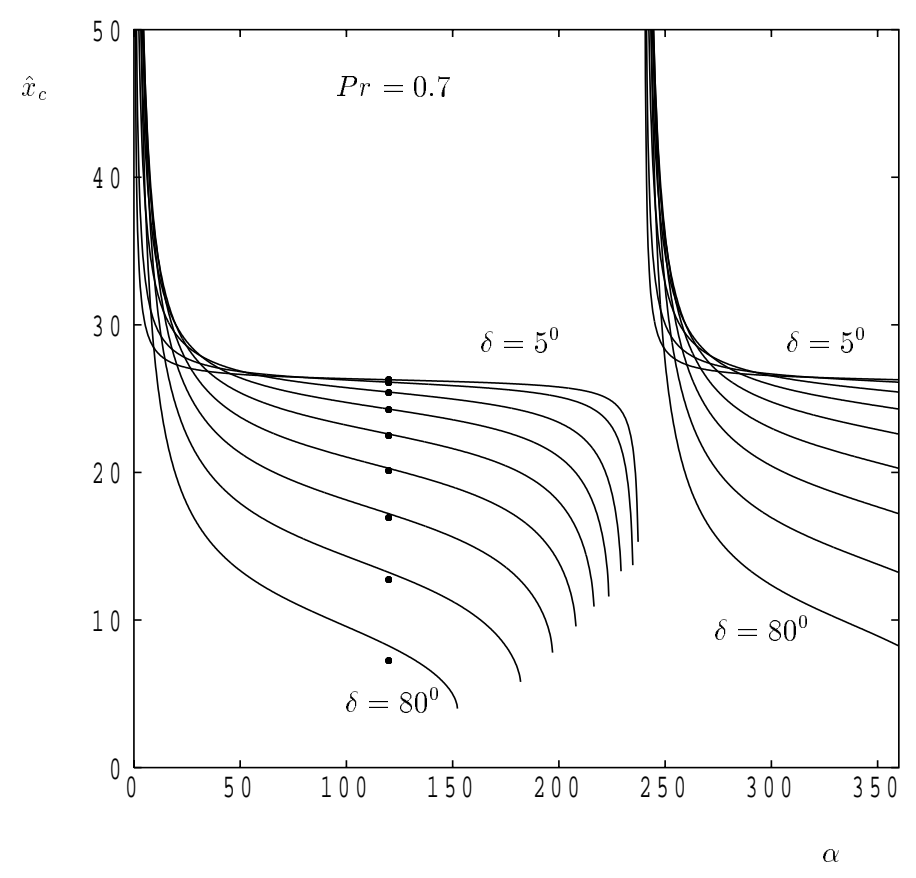

Fig. 3.

The variation of critical distance $x_{c}$ with wedge angle $\alpha$ for air $(\operatorname{Pr}=0.7)$ and $\delta=5^{0}, 10^{0}, 20^{\circ}, 30^{\circ}, 40^{\circ}$, $50^{\circ}, 60^{\circ}, 70^{0}, 80^{\circ}$. The dashed line denotes the corresponding result for the leading order boundary layer flow. 


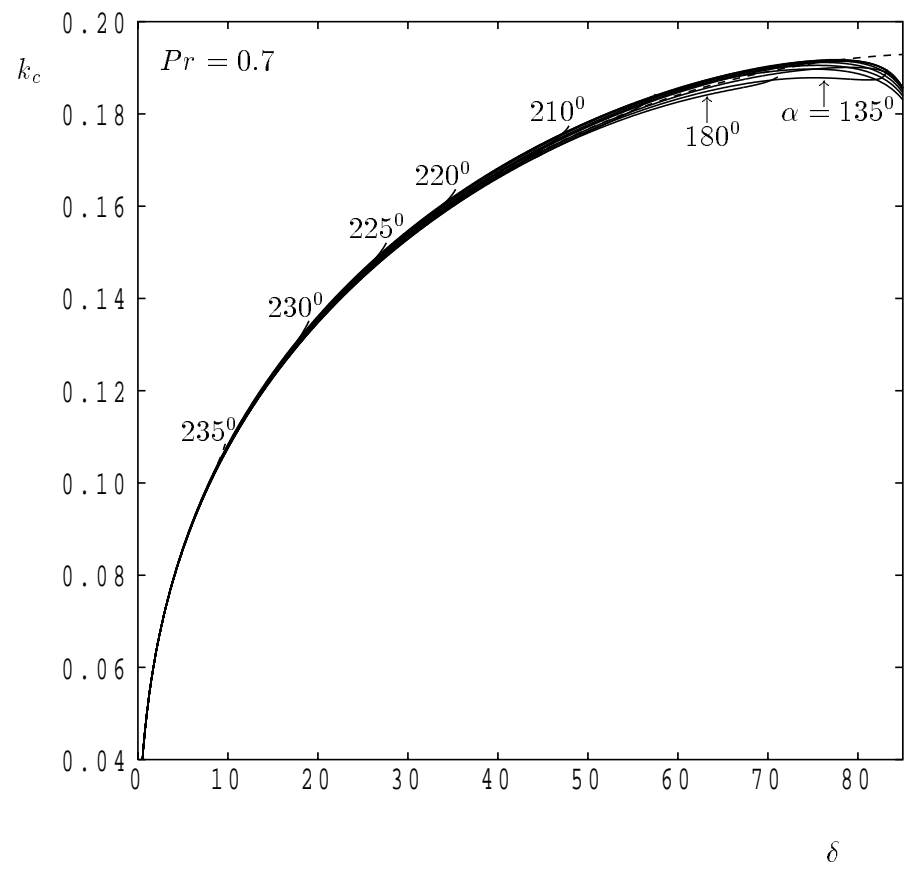

Fig. 4.

The variation of critical wavenumber $k_{c}$ with inclination angle $\delta$ for air $(P r=0.7)$ and $\alpha=5^{0}$ to $235^{0}$. The dashed line denotes the corresponding result for the leading order boundary layer flow. 


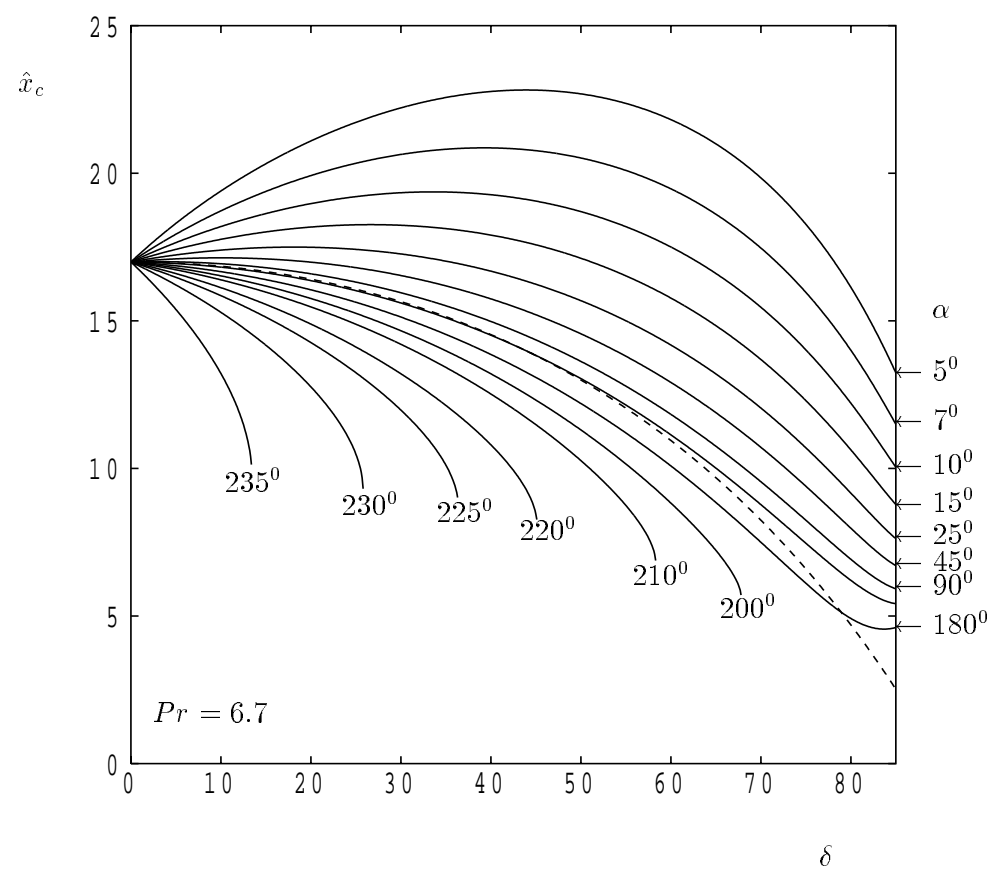

Fig. 5.

The variation of critical distance $\hat{x}_{c}$ with inclination angle $\delta$ for water $(\operatorname{Pr}=6.7)$ and $\alpha=5^{0}$ to $235^{0}$. The dashed line denotes the corresponding result for the leading order boundary layer flow. 


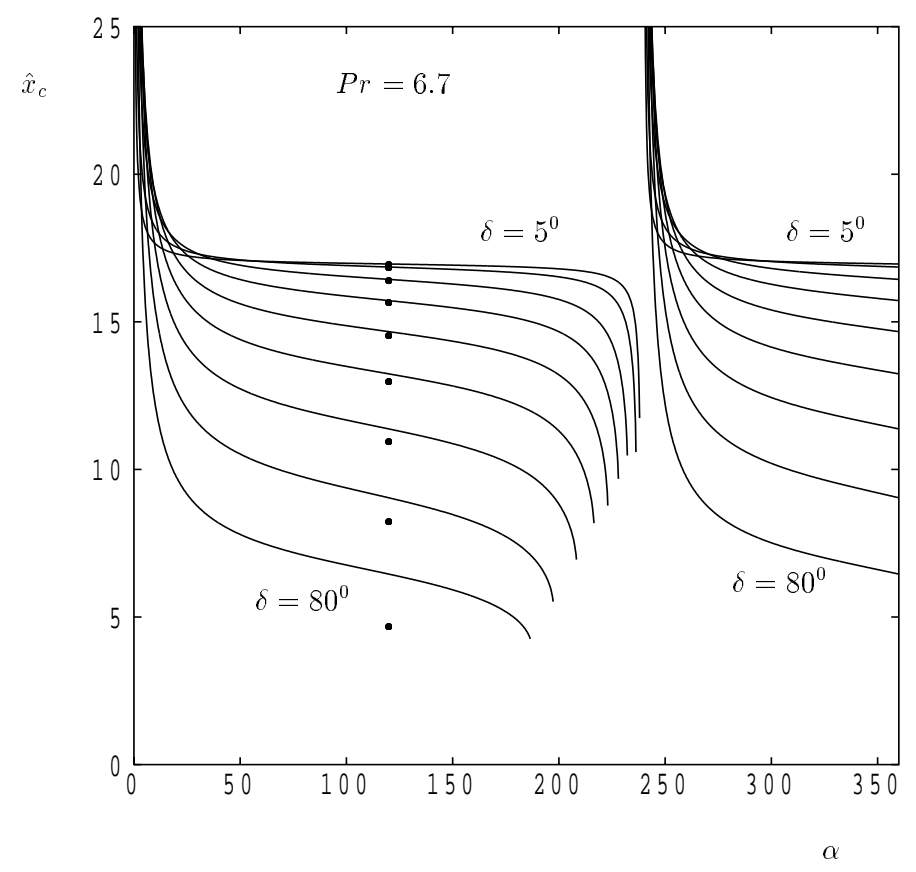

Fig. 6.

The variation of critical distance $x_{c}$ with wedge angle $\alpha$ for water $(\operatorname{Pr}=6.7)$ and $\delta=5^{0}, 10^{0}, 20^{0}, 30^{0}, 40^{\circ}$, $50^{\circ}, 60^{\circ}, 70^{\circ}, 80^{\circ}$. The dashed line denotes the corresponding result for the leading order boundary layer flow. 


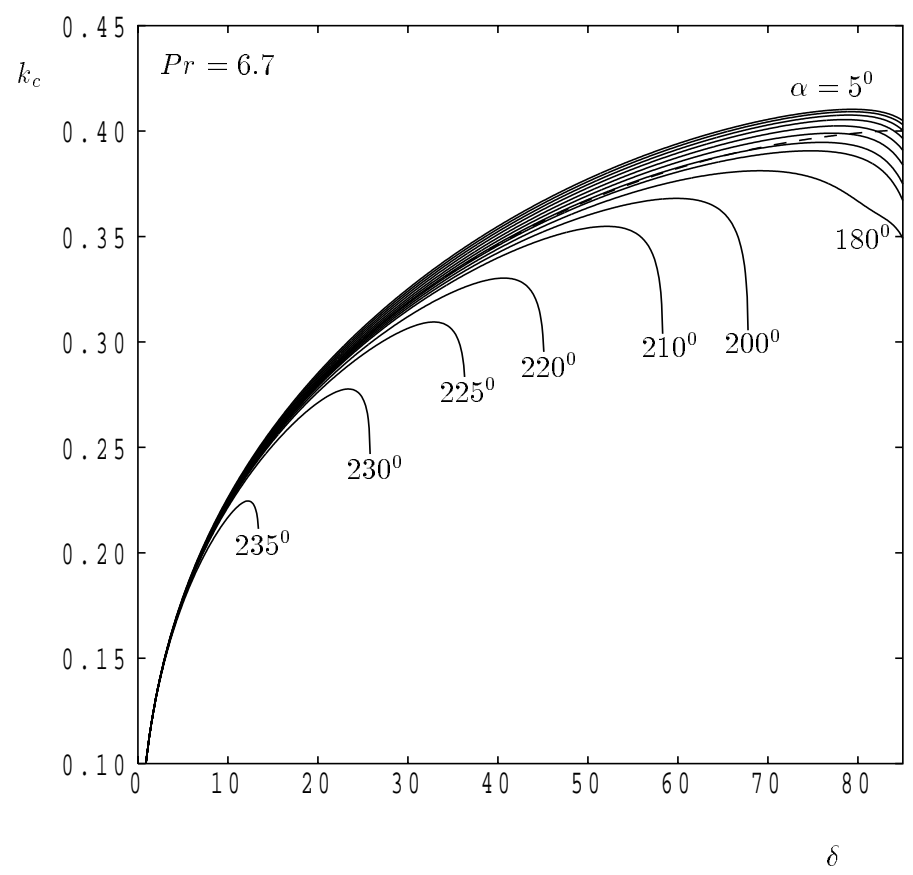

Fig. 7.

The variation of critical wavenumber $k_{c}$ with inclination angle $\delta$ for water $(P r=6.7)$ and $\alpha=5^{0}$ to $235^{0}$. The dashed line denotes the corresponding result for the leading order boundary layer flow. 